
\title{
3 Research Square \\ Inhibitory effects of aviptadil on the SARS-CoV-2 nsp10/ nsp16 protein complex
}

\section{Sultan F. Alnomasy ( $\nabla$ s.alnomasy@su.edu.sa )}

Department of Laboratory Science, College of Applied Medical Sciences, Alquwayiyah, Shaqra University

\section{Bader S. Alotaibi}

Department of Laboratory Science, College of Applied Medical Sciences, Alquwayiyah, Shaqra University

\section{Ziyad M. Aldosari}

Department of Laboratory Science, College of Applied Medical Sciences, Alquwayiyah, Shaqra University

\section{Ahmed H. Mujamammi}

Department of Pathology, Clinical Biochemistry, Unit, College of Medicine, King Saud University, Riyadh, Saudi Arabia

\section{Pragya Anand}

Babasaheb Bhimrao Ambedkar University

\section{Yusuf Akhter Akhter}

Babasaheb Bhimrao Ambedkar University

\section{Mohammad R. Hasan}

Department of Laboratory Science, College of Applied Medical Sciences, Alquwayiyah, Shaqra University

\section{Research Article}

\section{Keywords:}

Posted Date: February 16th, 2021

DOl: https://doi.org/10.21203/rs.3.rs-191980/v1

License: (c) (i) This work is licensed under a Creative Commons Attribution 4.0 International License. Read Full License 


\section{Inhibitory effects of aviptadil on the SARS-CoV-2 nsp10/ nsp16 protein complex}

Sultan F. Alnomasy ${ }^{1 *}$, Bader S. Alotaibi ${ }^{1}$, Ziyad M. Aldosari ${ }^{1}$, Ahmed H. Mujamammi ${ }^{2}$, Pragya Anand ${ }^{3}$, Yusuf Akhter ${ }^{3}$, Mohammad R. Hasan ${ }^{1 *}$

${ }^{1}$ Department of Medical Laboratory Sciences, College of Applied Medical Sciences, Alquwayiyah, Riyadh Province, Shaqra University, Saudi Arabia.

${ }^{2}$ Department of Pathology, Clinical Biochemistry Unit, College of Medicine, King Saud University, Riyadh, Saudi Arabia

${ }^{3}$ Department of Biotechnology, Babasaheb Bhimrao Ambedkar University, Vidya Vihar, Rae Bareli Road, Lucknow, India.

*Corresponding Authors: Email. 1. s.alnomasy@su.edu.sa and

2. malhasn@su.edu.sa 


\begin{abstract}
:
Severe acute respiratory syndrome coronavirus 2 (SARS-CoV-2), which emerged in late 2019, causes COVID-19, a disease that has been spreading rapidly worldwide. In human lung epithelial cells and monocytes, RLF-100 (aviptadil) has been found to inhibit the RNA replication machinery of SARS-CoV2, which includes several non-structural proteins (nsp) that play essential roles in synthesizing and replicating viral RNA. This virus is unique in requiring nsp10 and nsp16 for methyltransferase (MTase) activity. This enzyme is essential for RNA stability, protein translation, and viral ability to escape the host's immune recognition. Therefore, we aimed to use bioinformatics tools to analyze aviptadil's inhibitory effect on the SARS-CoV-2 nsp10/nsp16 complex. We present a comprehensive, in silicogenerated picture showing how aviptadil may interact with the nsp complex. Specifically, our model predicts how the initial binding of aviptadil to nsp10 and nsp16 may occur. This knowledge can assist drug development efforts against SARS-CoV-2 by providing more target information against nsp16.
\end{abstract}

\title{
Keywords
}

COVID-19, SARS-CoV-2, microbiology, aviptadil, coronavirus, in silico

\section{Introduction}

Severe acute respiratory syndrome coronavirus 2 (SARS-CoV-2) causes the disease known as COVID19, characterized by mild to severe respiratory illness, fever, and pneumonia. As of January 26, 2021, SARS-CoV-2 has infected approximately 100,350,064 persons with a mortality of 2,151,552 cases globally ${ }^{[1]}$. Thus far, no effective treatments are available, although vaccines against this coronavirus have been launched recently. The efficacy of these vaccines is currently being monitored. Three research approaches are currently being used to discover new drugs to combat the disease ${ }^{[2]:}$ The first approach examines current broad-spectrum anti-virals [3]; the second uses current molecular databases to find molecules that affect the coronavirus ${ }^{[4] ;}$ and the third approach essentially starts from scratch to develop new drugs based on various coronavirus genomic information ${ }^{[5]}$.

RLF-100 (aviptadil) is a synthetic form of the human vasoactive intestinal polypeptide (VIP) and pituitary adenylate cyclase-activating polypeptide (PACAP) that can inhibit the replication of SARS-CoV-2 in lung epithelial cells and monocytes ${ }^{[6] . ~ A v i p t a d i l ~ h a s ~ b e e n ~ d e s i g n a t e d ~ b y ~ t h e ~ F D A ~ a s ~ a n ~ o r p h a n ~ d r u g ~ t o ~}$ treat ARDS, pulmonary hypertension, and SARS-CoV-2; and it has been granted Fast Track Designation to treat ARDS/acute lung injury in COVID-19 [7-8]. VIP and PACAP are neuropeptides that play roles in regulating the human intestinal and immune systems ${ }^{[9] .}$ VIP, a 28 -amino acid peptide ${ }^{[10]}$, was isolated 
from the hog intestine in 1970 [11], while PACAP, a 38-amino acid peptide, was isolated from the ovine hypothalamus in $1989^{[12] .}$. These peptides act to increase the production of cyclic AMP in cells ${ }^{[13] .}$

The SARS-CoV-2 RNA replication machinery includes several non-structural proteins (nsp) that play a vital role in the synthesis and replication of the viral RNA. The nsp7-nsp16 complex is involved in viral RNA capping ${ }^{[14]}$. Specifically, the SARS-CoV-2 nsp16/nsp10 complex works as a 2'-Omethyltransferase (MTase) ${ }^{[15]}$. This nsp10/nsp16 complex is necessary to evade immune recognition ${ }^{[16-}$

${ }^{17]}$. Here, we present an in silico analysis of the interaction between aviptadil and SARS-CoV-2 nsp16 to generate new information that can be used to target the virus. Our overall aim is to apply a computational approach to provide more target information that can be used for drug intervention against SARS-CoV-2.

\section{Results}

\section{Interaction model of docked RLF-100 (aviptadil) with the SARS-CoV-2 nsp16/nsp10 complex}

We searched the PDB database for the three-dimensional structure of the nsp16/nsp10 complex, and found accession number $6 \mathrm{~W} 4 \mathrm{H}$. The $6 \mathrm{~W} 4 \mathrm{H}$ structure has been elucidated by X-ray diffraction at a resolution of $1.80 \AA$ and shows a complex composed of nsp16 and nsp10. This heterodimer complex is necessary to cap the viral mRNA transcripts for the effective translation of coronavirus RNA ${ }^{[18]}$. The nsp10/nsp16 complex is also essential for preventing immune recognition ${ }^{[16-17]}$.

This work identified the binding sites of aviptadil on nsp16 of SARS-CoV-2using the GalaxyPepDock server to dock aviptadil within nsp16 (PDBID_6W4H). The amino acid sequence of aviptadil (HSDAVFTDNYTRLRKQMAVKKYLNSILN) was submitted to the server in FASTA format. The top model was selected by aligning the $\mathrm{x}$ and calculating their protein structural similarity $(\mathbf{T M}$ score $=$ 0.570) with more weight attached to interacting residues. The accuracy of $x(0.213)$ was calculated using a modified version of BLOSUM62. The protein-peptide interaction of the selected model was visualized via PyMol v2.4.1, and the results are shown in Figure 1. The docking structure was further analyzed using PDBsum Generate (http://www.ebi.ac.uk/thornton-srv/databases/pdbsum/Generate.html), and the results are shown in Figure 2A. The output from the PDBsum was processed by DIMPLOT v2.2 to generate a two-dimensional view of the interactions between $\mathrm{x}$ as shown in Figure $2 \mathrm{C}^{[19-20]}$. The three-dimensional view of the interaction was also generated by PyMol and is shown in Figure 3.

\section{Discussion}

The interaction between nsp10 and nsp16 of SARS-CoV has been reported previously ${ }^{[15,20-21,23]}$, with yeast and mammalian two-hybrid systems being used to map this interaction ${ }^{[21,23]}$. The three-dimensional structure generated in this study also shows the essential interactions between nsp10 and nsp16 (Figure 1D). The structure shows significant contact areas across amino acid residues Asn40-Thr47, Val57-Pro59, 
Gly69-Ser72, Cys77-Pro84, Lys93-Tyr96 of nsp10 (Figure 1D). These results agree well with previous reports on the interaction domain of the SARS coronavirus ${ }^{[15,21-23] . ~ I n ~ a d d i t i o n, ~ K e, ~ 2012, ~}{ }^{[24]}$ showed that the area between Gln65 and Pro107 is vital for its interaction with nsp16, while the region between Val42 and Cys120 of nsp10 is a vital area for the complete enzymatic activity of nsp16. Our results show the interaction between the aviptadil peptide and the nsp16/nsp10 protein complex. Specifically, the Chain A (blue)-Chain C (red) polar contacts involve the following amino acid residues: Asn299-Ser440, Val297-Asn443, Gly149-Tyr437, Gln159-Lys430, Asn178-Arg429, Ser146-Arg429, Ser146-Arg429, Lys147-Arg429, Asr221-Thr422, Lys183-Asp423, Lys183-Asp423, and Gln219-Asp423. The results of PDBsum analysis (Figure 2A) show circular areas representing the surface area of the protein, where Chains A (violet), B (red), and C (dark yellow) are shown with decreasing surface areas, respectively. The interaction between Chains $\mathrm{A}$ and $\mathrm{C}$ is of particular interest, and Figure $2 \mathrm{~B}$ shows the extended interface regions in the circles that indicate the respective chains interacting with each other; and the shaded area indicate the interface area of the interacting chain. The model shows a total of 28 residues of Chain A and 18 residues of Chain $\mathrm{C}$ interacting with each other, where red lines indicate 2 salt bridges, blue lines indicate 10 hydrogen bonds, and orange lines indicate 136 non-bonded interactions. The 10 hydrogen bonds between Chains A and C involve the following amino acid residues: Gly149-Tyr437, Val297Asn443, Lys147-Arg429, Ser146-Arg429, Ans178-Arg429, Asn299-Ser440, Lys183-Asp423, and GIn219-Asp423, which are listed in Table 1. DIMPLOT was used to visualize the two-dimensional protein-protein interaction between aviptadil and Chain A of SARS- CoV-2 nsp16 protein (Figure 2C).

\section{Conclusion}

The results of structural bioinformatics analyses indicate the potential sites of binding specificity between aviptadil and nsp16. The model predicts how the initial binding of aviptadil with nsp10 and nsp16 may occur. Moreover, this binding may inhibit the 2 -O-MTase activity of the SARS-CoV nsp10/16 complex.

\section{Methods}

\section{SARS-CoV-2 nsp16 interaction with the aviptadil (RLF-100) peptide}

The tertiary structure of the target protein (PDBID_6W4H) comprising the nsp16-nsp10 complex of SARS-CoV-2 was obtained from the PDB database. Likewise, the peptide sequence for aviptadil was retrieved from the PDB database [25]. Docking was performed using the GalaxyPepDock server (http://galaxy.seoklab.org/cgi-bin/submit.cgi?type=PEPDOCK), which does not require prior binding site information and predicts $\mathrm{x} 75.4 \%$ residues ${ }^{[26] .}$ It requires a PDB file and FASTA sequence as input. Protein-peptide docking was predicted based on interaction similarity. The required input is the target protein in PDB format $(6 \mathrm{~W} 4 \mathrm{H})$ and the peptide sequence of aviptadil [27] 
(HSDAVFTDNYTRLRKQMAVKKYLNSILN) in FASTA format. GalaxyPepDock's protein and peptide sizes are limited to 900 and 30 amino acids, respectively. It performs a flexible-structure, energybased optimization using a global docking approach. It does not require prior binding site information and also generates protein structure similarity scores. Its predictions are based on structural alignments with the PepBind database and interaction similarity. It uses Galaxy TBM as its model building tool for protein structural alignments; and peptide sequences are aligned using a modified BLOSUM62 matrix score. Finally, it also refines the protein structure using Galaxy energy. This is accomplished by adjusting the backbone of the structure of the complex and rebuilding the side chains. repackaging done. We relaxed the overall structure of the complex by simulating the molecular dynamics [28-29,]. The docked complex was visualized using PyMol 2.4.1, which also generated the output of interaction analysis [30].

Acknowledgements: We thank Shaqra University for the facility.

Author contributions: SA, Conceived the idea, analyzed the results, wrote the manuscript, BA, ZA, AM, reviewed the manuscript, PA, YA, MH, did the bioinformatics analysis, reviewed and finalized the manuscript.

\section{Competing interests: None declared}

\section{Data availability: Supplementary file attached}

Ethics declarations: this work has not been submitted or accepted for publication in another journal or book. All authors have approved its submission for publication, and all persons entitled to authorship have been so named. 


\section{References}

1. WHO Coronavirus Disease (COVID-19) Dashboard 2021 https://covid19.who.int/

2. Zumla, Alimuddin, Chan, Jasper F. W., Azhar, Esam I., Hui, David S. C., \& Yuen, Kwok-Yung. (2016). Coronaviruses - drug discovery and therapeutic options. Nature Reviews Drug Discovery, 15(5), 327-347. doi: 10.1038/nrd.2015.37

3. Chan, Jasper F. W., Chan, Kwok-Hung, Kao, Richard Y. T., To, Kelvin K. W., Zheng, Bo-Jian, Li, Clara P. Y., Yuen, Kwok-Yung. (2013). Broad-spectrum antivirals for the emerging Middle East respiratory syndrome coronavirus. Journal of Infection, 67(6), 606-616. doi: https://doi.org/10.1016/j.jinf.2013.09.029

4. Dyall, Julie, Coleman, Christopher M., Hart, Brit J., Venkataraman, Thiagarajan, Holbrook, Michael R., Kindrachuk, Jason, Frieman, Matthew B. (2014). Repurposing of Clinically Developed Drugs for Treatment of Middle East Respiratory Syndrome Coronavirus Infection. Antimicrobial Agents and Chemotherapy, 58(8), 4885. doi: 10.1128/AAC.03036-14

5. Omrani, Ali S., Saad, Mustafa M., Baig, Kamran, Bahloul, Abdelkarim, Abdul-Matin, Mohammed, Alaidaroos, Amal Y., Albarrak, Ali M. (2014). Ribavirin and interferon alfa-2a for severe Middle East respiratory syndrome coronavirus infection: a retrospective cohort study. The Lancet Infectious Diseases, 14(11), 1090-1095. doi: https://doi.org/10.1016/S14733099(14)70920-X

6. Temerozo, Jairo R., Sacramento, Carolina Q., Fintelman-Rodrigues, Natalia, Pão, Camila R. R., de Freitas, Caroline S., da Silva Gomes Dias, Suelen, . . Bou-Habib, Dumith Chequer. (2020). The neuropeptides VIP and PACAP inhibit SARS-CoV-2 replication in monocytes and lung epithelial cells, decrease production of proinflammatory cytokines, and VIP levels are associated with survival in severe Covid-19 patients. bioRxiv, 2020.2007.2025.220806. doi: 10.1101/2020.07.25.220806

7. Zheng, B., Guan, Y., Huang, J., \& He, M. L. (2009). U.S. Patent No. 7,491,489. Washington, DC: U.S. Patent and Trademark Office.

8. Sarkar, C., Mondal, M., Torequl Islam, M., Martorell, M., Docea, A. O., Maroyi, A., ... \& Calina, D. (2020). Potential therapeutic options for COVID-19: current status, challenges, and future perspectives. Frontiers in pharmacology, 11, 1428.

9. Moody, T. W., Ito, T., Osefo, N., \& Jensen, R. T. (2011). VIP and PACAP: recent insights into their functions/roles in physiology and disease from molecular and genetic studies. Curr Opin Endocrinol Diabetes Obes, 18(1), 61-67. doi: 10.1097/MED.0b013e328342568a

10. Bacher, G., \& Bevec, D. (2012). U.S. Patent No. 8,178,489. Washington, DC: U.S. Patent and Trademark Office.

11. Said, S. I., \& Mutt, V. (1970). Polypeptide with broad biological activity: isolation from small intestine. Science, 169(3951), 1217-1218.

12. Miyata, A., Arimura, A., Dahl, R. R., Minamino, N., Uehara, A., Jiang, L., Coy, D. H. (1989). Isolation of a novel 38 residue-hypothalamic polypeptide which stimulates adenylate cyclase in pituitary cells. Biochem Biophys Res Commun, 164(1), 567-574.

13. Harmar, A. J., Fahrenkrug, J., Gozes, I., Laburthe, M., May, V., Pisegna, J. R., Said, S. I. (2012). Pharmacology and functions of receptors for vasoactive intestinal peptide and pituitary adenylate cyclase-activating polypeptide: IUPHAR review 1. Br J Pharmacol, 166(1), 4-17. doi: 10.1111/j.1476-5381.2012.01871.x

14. Chen, Y., Cai, H., Pan, J., Xiang, N., Tien, P., Ahola, T., \& Guo, D. (2009). Functional screen reveals SARS coronavirus nonstructural protein nsp14 as a novel cap N7 methyltransferase. Proc Natl Acad Sci U S A, 106(9), 3484-3489. doi: 10.1073/pnas.0808790106

15. Bouvet, M., Debarnot, C., Imbert, I., Selisko, B., Snijder, E. J., Canard, B., \& Decroly, E. (2010). In vitro reconstitution of SARS-coronavirus mRNA cap methylation. PLoS Pathog, 6(4), e1000863. doi: 10.1371/journal.ppat.1000863 
16. Bollati, M., Milani, M., Mastrangelo, E., Ricagno, S., Tedeschi, G., Nonnis, S., Bolognesi, M. (2009). Recognition of RNA cap in the Wesselsbron virus NS5 methyltransferase domain: implications for RNA-capping mechanisms in Flavivirus. J Mol Biol, 385(1), 140-152. doi: 10.1016/j.jmb.2008.10.028

17. von Grotthuss, Marcin, Wyrwicz, Lucjan S., \& Rychlewski, Leszek. (2003). mRNA cap-1 methyltransferase in the SARS genome. Cell, 113(6), 701-702. doi: 10.1016/s00928674(03)00424-0

18. Chen, Yu, \& Guo, Deyin. (2016). Molecular mechanisms of coronavirus RNA capping and methylation. Virologica Sinica, 31(1), 3-11. doi: 10.1007/s12250-016-3726-4

19. Laskowski, R. A., Jabłońska, J., Pravda, L., Vařeková, R. S., \& Thornton, J. M. (2018). PDBsum: Structural summaries of PDB entries. Protein science, 27(1), 129-134.

20. Laskowski, R. A., \& Swindells, M. B. (2011). LigPlott: multiple ligand-protein interaction diagrams for drug discovery.

21. Imbert, Isabelle, Snijder, Eric J., Dimitrova, Maria, Guillemot, Jean-Claude, Lécine, Patrick, \& Canard, Bruno. (2008). The SARS-Coronavirus PLnc domain of nsp3 as a replication/transcription scaffolding protein. Virus Research, 133(2), 136-148. doi: https://doi.org/10.1016/j.virusres.2007.11.017

22. Chen, Yu, Su, Ceyang, Ke, Min, Jin, Xu, Xu, Lirong, Zhang, Zhou, Guo, Deyin. (2011). Biochemical and structural insights into the mechanisms of SARS coronavirus RNA ribose 2'-Omethylation by nsp16/nsp10 protein complex. PLoS pathogens, 7(10), e1002294-e1002294. doi: 10.1371/journal.ppat.1002294

23. Pan, Ji'an, Peng, Xiaoxue, Gao, Yajing, Li, Zhilin, Lu, Xiaolu, Chen, Yingzhao, ... Guo, Deyin. (2008). Genome-wide analysis of protein-protein interactions and involvement of viral proteins in SARS-CoV replication. PloS one, 3(10), e3299-e3299. doi: 10.1371/journal.pone.0003299

24. Ke, Min, Chen, Yu, Wu, Andong, Sun, Ying, Su, Ceyang, Wu, Hao, Guo, Deyin. (2012). Short peptides derived from the interaction domain of SARS coronavirus nonstructural protein nsp10 can suppress the 2'-O-methyltransferase activity of nsp10/nsp16 complex. Virus research, 167(2), 322-328. doi: 10.1016/j.virusres.2012.05.017

25. Gross, P.C, Burkart, S.C; Müller, R. Analytics of the therapeutic peptide aviptadil by sheathless CE-MS and comparison with nanoRP-HPLC-MS. Journal of Pharmaceutical and Biomedical Analysis, 2014, Volume 88, 25 January 2014, Pages 477-482

26. Ciemny, M., Kurcinski, M., Kamel, K., Kolinski, A., Alam, N., Schueler-Furman, O., \& Kmiecik, S. (2018). Protein-peptide docking: opportunities and challenges. Drug discovery today, 23(8), 1530-1537.

27. Junior N, J. A. C., Santos, A. M., Quintans-Júnior, L. J., Walker, C. I. B., Borges, L. P., \& Serafini, M. R. (2020). SARS, MERS and SARS-CoV-2 (COVID-19) treatment: a patent review. Expert Opinion on Therapeutic Patents, (just-accepted).

28. Lee, H., Heo, L., Lee, M. S., \& Seok, C. (2015). GalaxyPepDock: a protein-peptide docking tool based on interaction similarity and energy optimization. Nucleic acids research, 43(W1), W431W435.

29. Heo, L., Park, H., \& Seok, C. (2013). GalaxyRefine: protein structure refinement driven by sidechain repacking. Nucleic acids research, 41(W1), W384-W388.

30. DeLano, W. L. (2002). Pymol: An open-source molecular graphics tool. CCP4 Newsletter on protein crystallography, 40(1), 82-92. 
Table 1: Residues involved in hydrogen bond interactions between Chains $A$ and $C$

\begin{tabular}{ccccc}
\hline $\begin{array}{c}\text { Chain A } \\
\text { residues }\end{array}$ & Atom type & Distance $(\AA)$ & $\begin{array}{c}\text { Chain C } \\
\text { residues }\end{array}$ & Atom type \\
\hline $\mathrm{SER}^{146}$ & $\mathrm{O}$ & $2.88 \AA$ & $\mathrm{ARG}{ }^{429}$ & $\mathrm{NH} 2$ \\
$\mathrm{SER}^{146}$ & $\mathrm{OG}$ & $2.84 \AA$ & $\mathrm{ARG}^{429}$ & $\mathrm{NH} 1$ \\
$\mathrm{LYS}^{147}$ & $\mathrm{O}$ & $2.96 \AA$ & $\mathrm{ARG}^{429}$ & $\mathrm{NH} 1$ \\
$\mathrm{GLY}^{149}$ & $\mathrm{~N}$ & $2.99 \AA$ & $\mathrm{TYR}^{437}$ & $\mathrm{OH}$ \\
$\mathrm{ASN}^{178}$ & $\mathrm{OD} 1$ & $2.99 \AA$ & $\mathrm{ARG}^{429}$ & $\mathrm{NH} 1$ \\
$\mathrm{LYS}^{183}$ & $\mathrm{NZ}$ & $2.78 \AA$ & $\mathrm{ASP}^{423}$ & $\mathrm{OD} 1$ \\
$\mathrm{LYS}^{183}$ & $\mathrm{NZ}$ & $2.82 \AA$ & $\mathrm{ASP}^{423}$ & $\mathrm{OD} 2$ \\
$\mathrm{GLN}^{219}$ & $\mathrm{NE} 2$ & $2.94 \AA$ & $\mathrm{ASP}^{423}$ & $\mathrm{OD} 2$ \\
$\mathrm{VAL}^{297}$ & $\mathrm{~N}$ & $3.09 \AA$ & $\mathrm{ASN}^{443}$ & $\mathrm{OD} 1$ \\
$\mathrm{ASN}^{299}$ & $\mathrm{ND} 2$ & $2.90 \AA$ & $\mathrm{SER}^{440}$ & $\mathrm{O}$ \\
\hline
\end{tabular}

\section{Figure Legends:}

Figure 1: Overall three-dimensional view of interactions between the structural complex of SARS-CoV-2 nsp10/nsp16 and aviptadil peptide. (A) Docking model of the surface interaction between aviptadil with SARS-CoV-2 nsp16. (B) Three-dimensional structure of aviptadil (red). (C) Three-dimensional structure of nsp16 (blue). (D) Docking of aviptadil within the SARS-CoV-2 nsp10 (cyan)/nsp16 (blue) complex. This model of aviptadil- nsp16 interaction was produced using GalaxyPepDock.

Figure 2: Interactions study between the chains (A) Chain A (nsp16 in violet), Chain B (nsp10 in red) and Chain $\mathrm{C}$ (aviptadil in dark yellow), where circular areas represent the surface areas of the protein chains; and non-bonded interactions, i.e., hydrogen bonds and salt bridges, are indicated as orange, light blue, and red lines, respectively. (B) In Chains A and B, 24 and 18 residues, respectively, are interacting through various bonds. (C) 2D representation of the interaction between Chains A and C, indicating residues of particular chains, bonds (H-bonds in light blue and salt bridges in red), and bond lengths. The black dotted line indicates interphase between chains.

Figure 3: Three-dimensional structure of the complex between Nsp16 (blue) and aviptadil (red). Yellow dashes indicate hydrogen bonds between respective atoms of inter-chains. 
Figures 1.

(A) Docking model of SARS-C 0V-2 target protein interaction with peptide chain

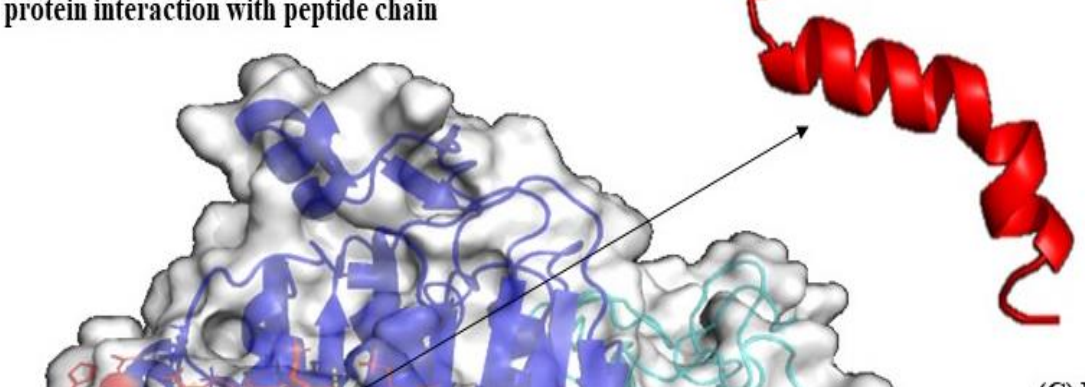

(D) Protein-Peptide complex

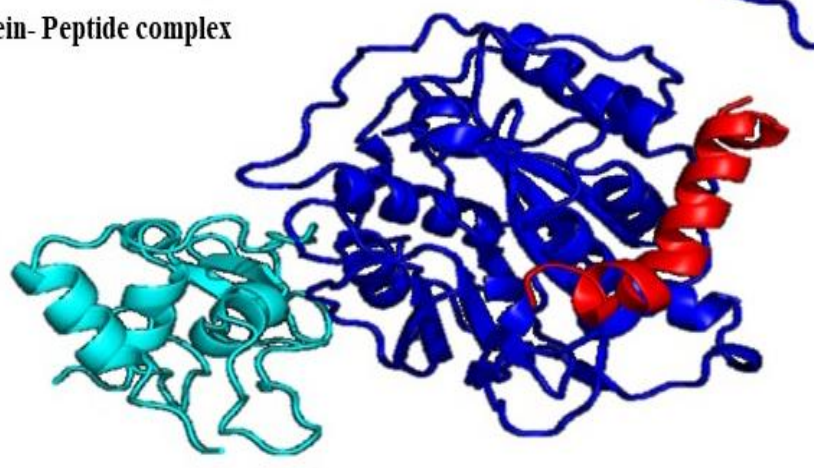

(C) Non- Structural Protein -16

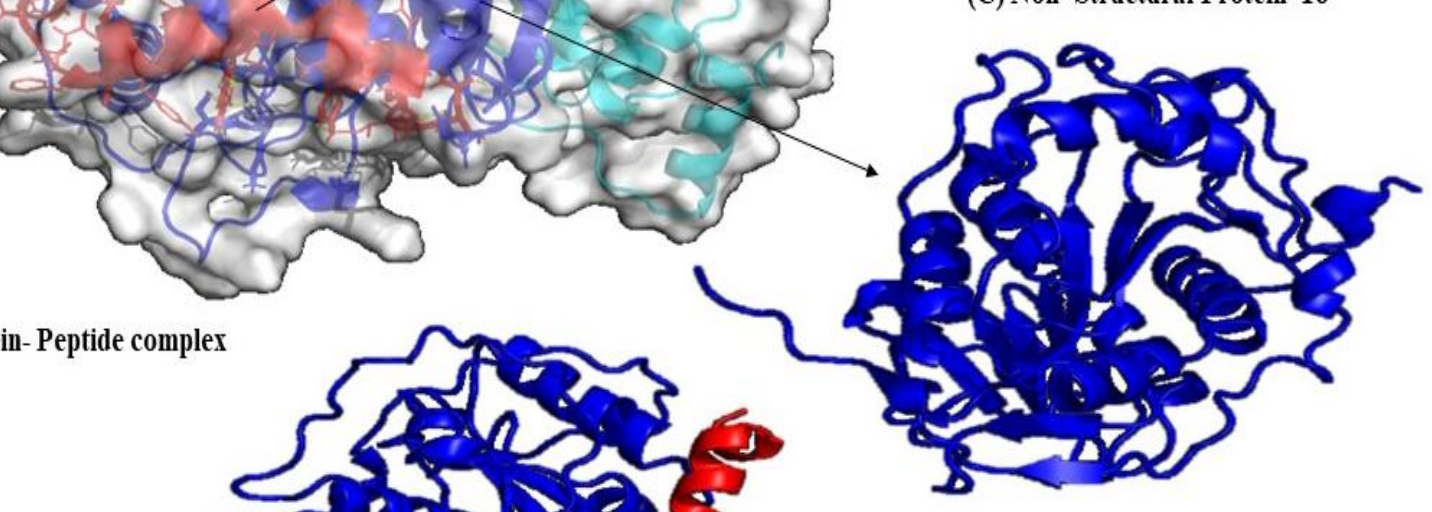


Figures 2.

(A) Interaction between
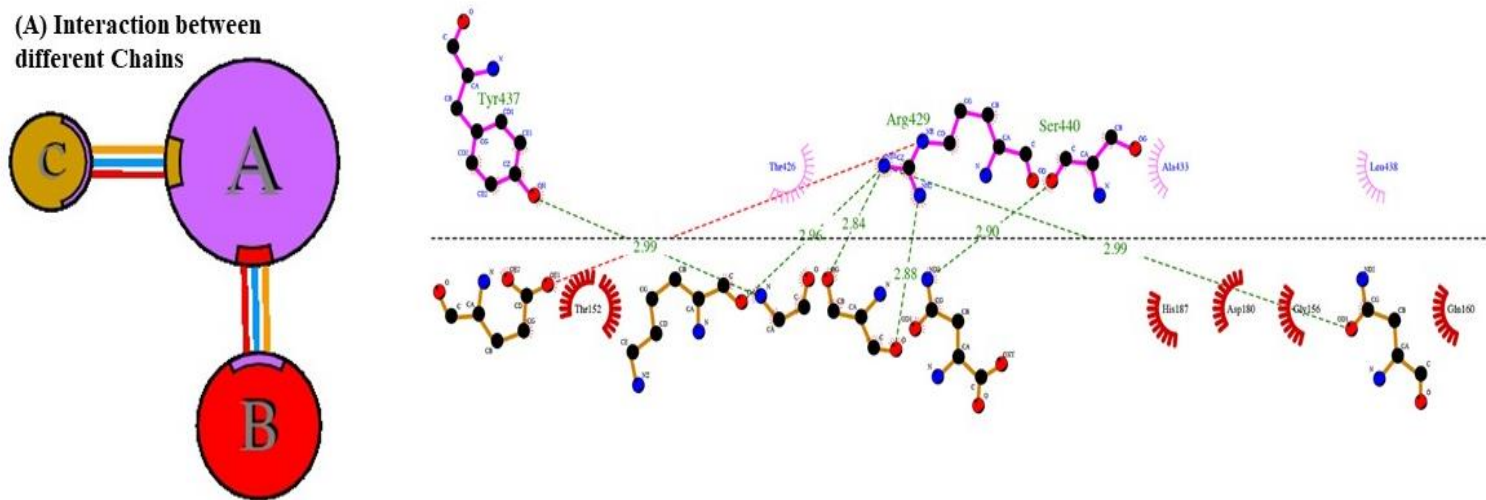

Chain A Chain C

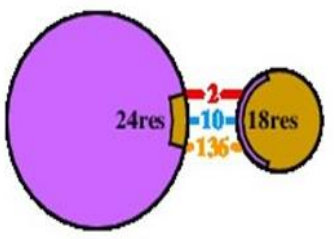

(B) Chain A and Chain C interaction and residues involved

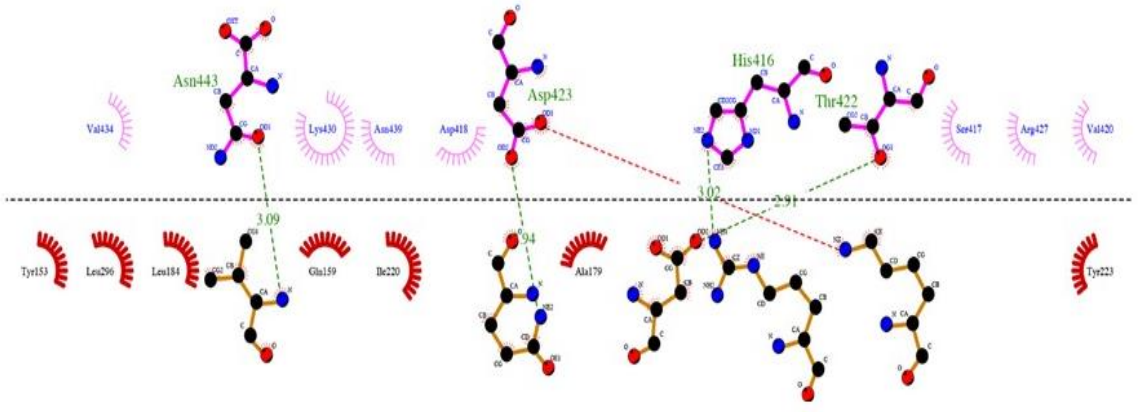

(C) Two dimensional representation of interaction study 
Figures 3.

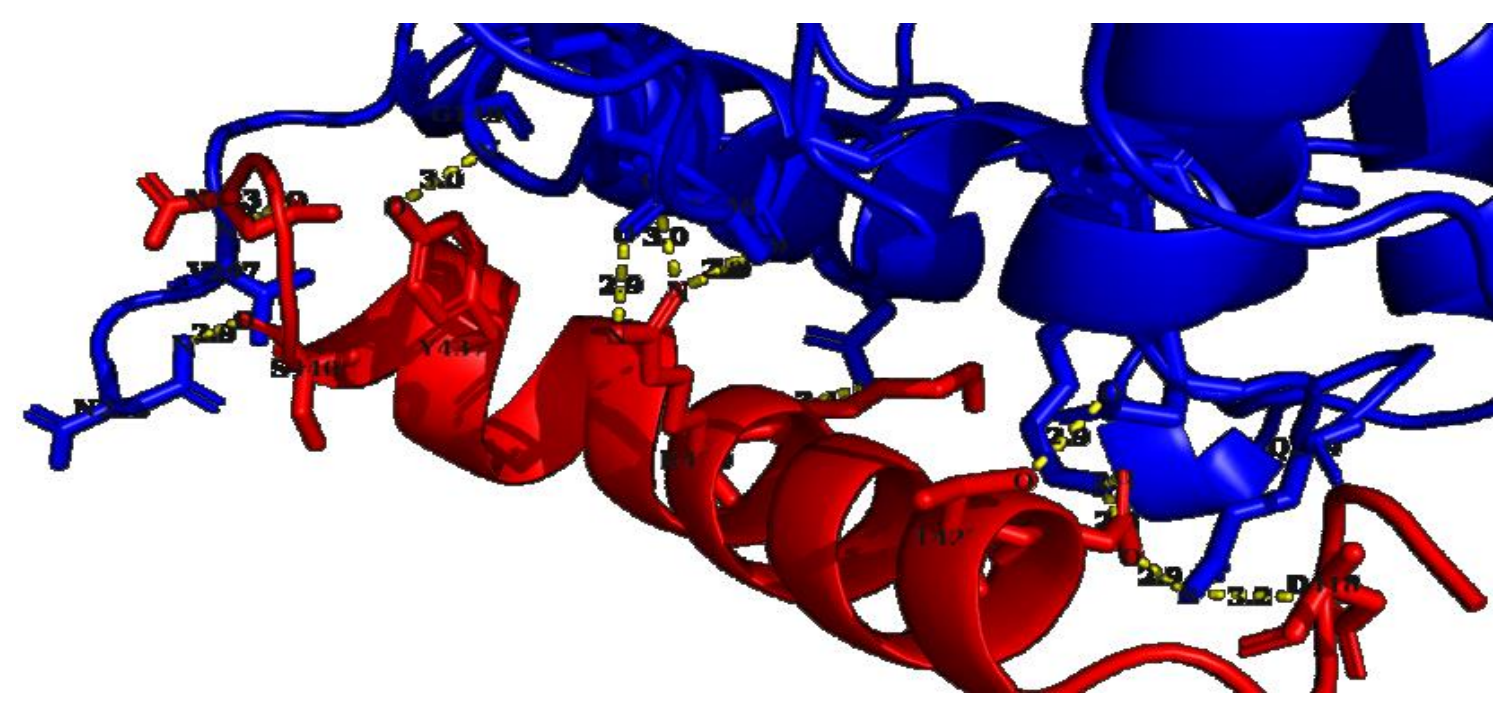

Page 11 of 11 


\section{Figures}

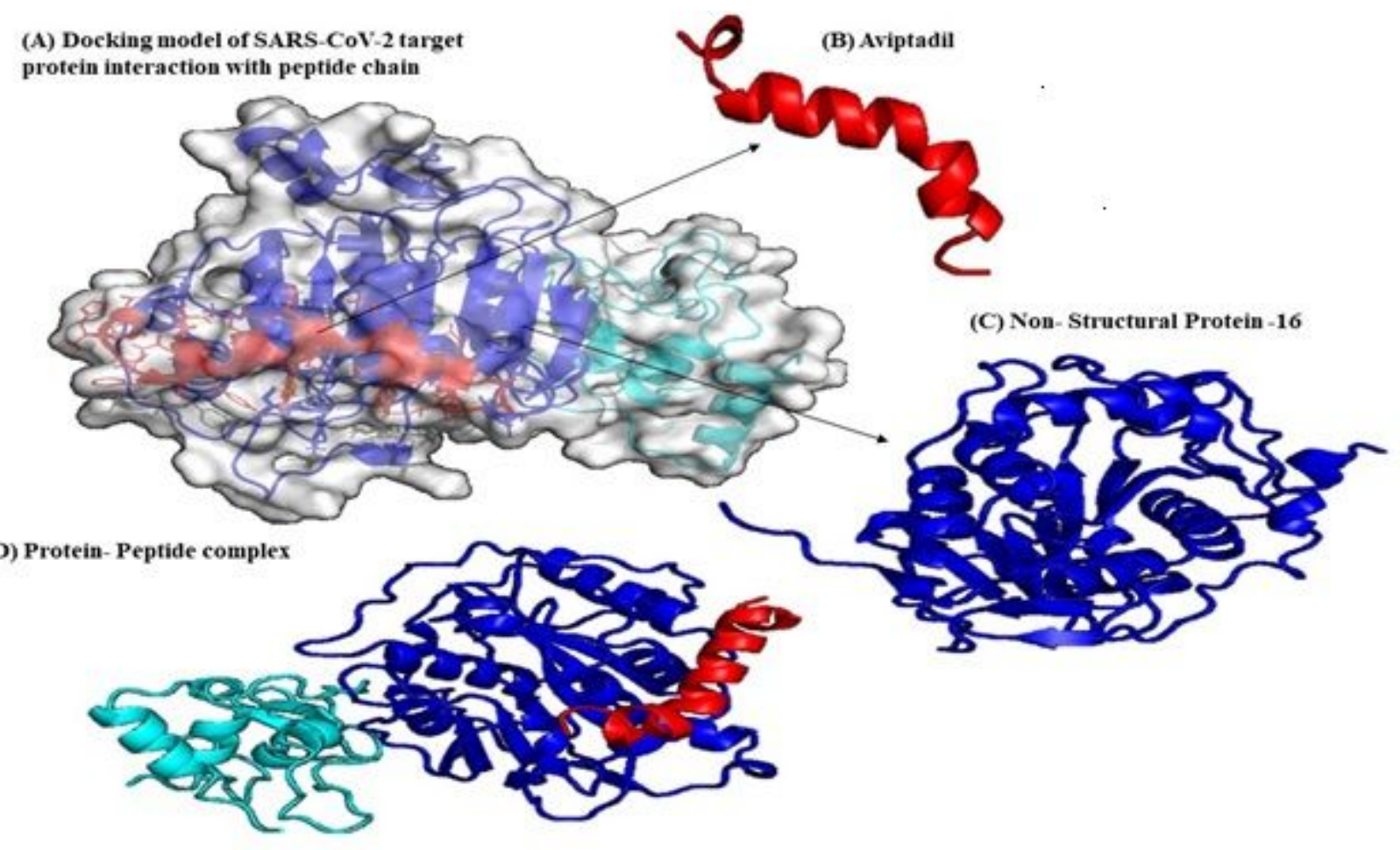

\section{Figure 1}

Overall three-dimensional view of interactions between the structural complex of SARS-CoV-2 nsp10/nsp16 and aviptadil peptide. (A) Docking model of the surface interaction between aviptadil with SARS-CoV-2 nsp16. (B) Three-dimensional structure of aviptadil (red). (C) Three-dimensional structure of nsp16 (blue). (D) Docking of aviptadil within the SARS-CoV-2 nsp10 (cyan)/nsp16 (blue) complex. This model of aviptadil- nsp16 interaction was produced using GalaxyPepDock. 


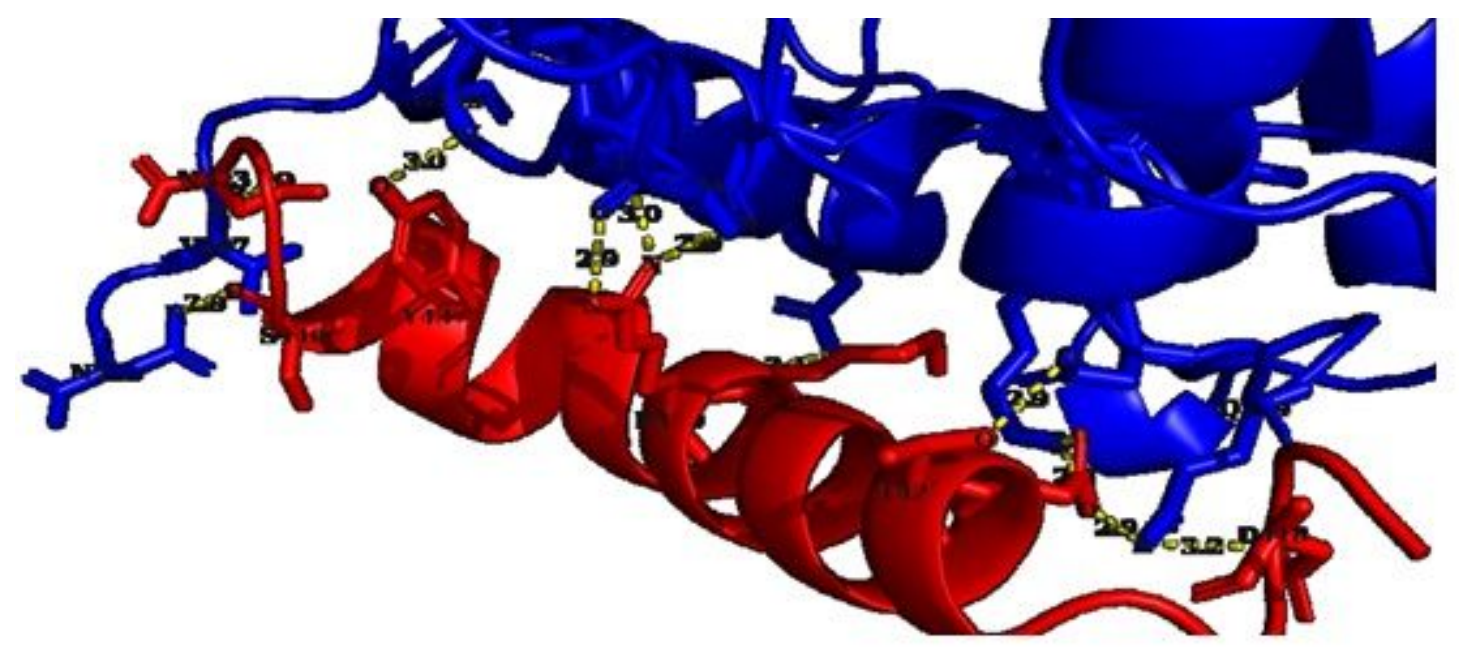

Figure 2

Interactions study between the chains (A) Chain A (nsp16 in violet), Chain B (nsp10 in red) and Chain C (aviptadil in dark yellow), where circular areas represent the surface areas of the protein chains; and nonbonded interactions, i.e., hydrogen bonds and salt bridges, are indicated as orange, light blue, and red lines, respectively. (B) In Chains A and B, 24 and 18 residues, respectively, are interacting through various bonds. (C) $2 D$ representation of the interaction between Chains $A$ and $C$, indicating residues of particular chains, bonds ( $\mathrm{H}$-bonds in light blue and salt bridges in red), and bond lengths. The black dotted line indicates interphase between chains. 
(A) Interaction between
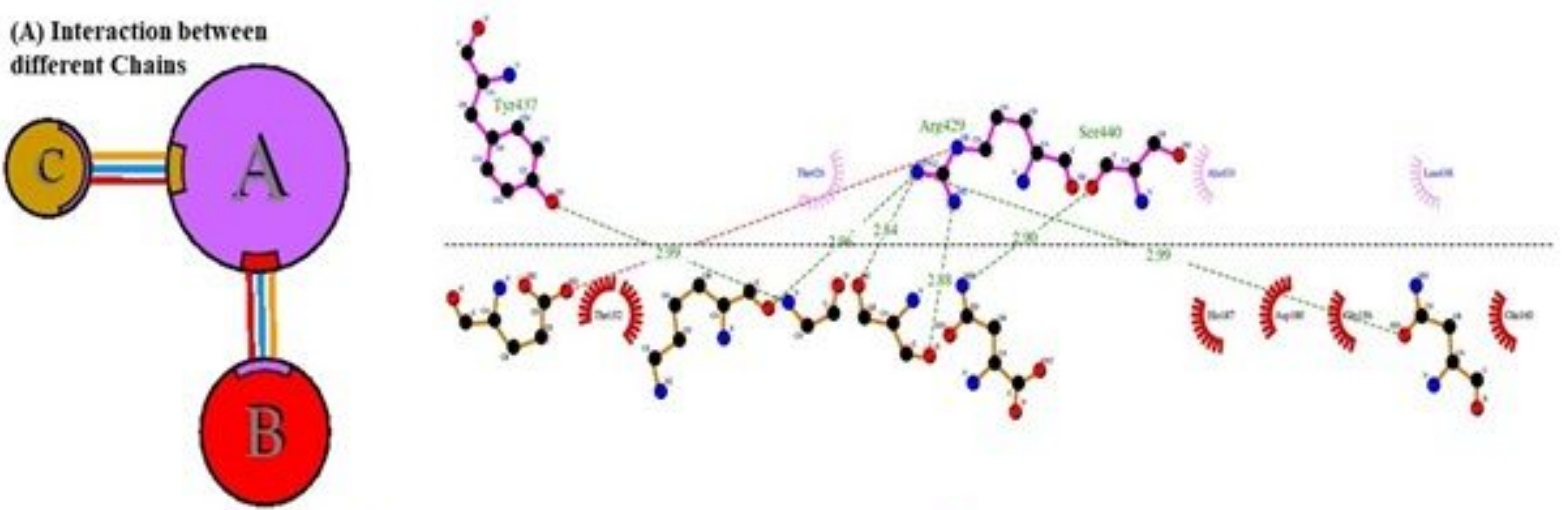

Chain A Chain C

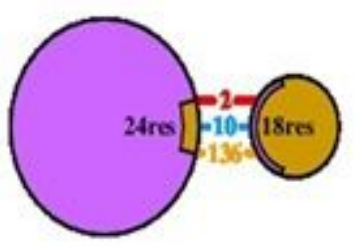

(B) Chain $\mathrm{A}$ and Chain C interaction and residues involved

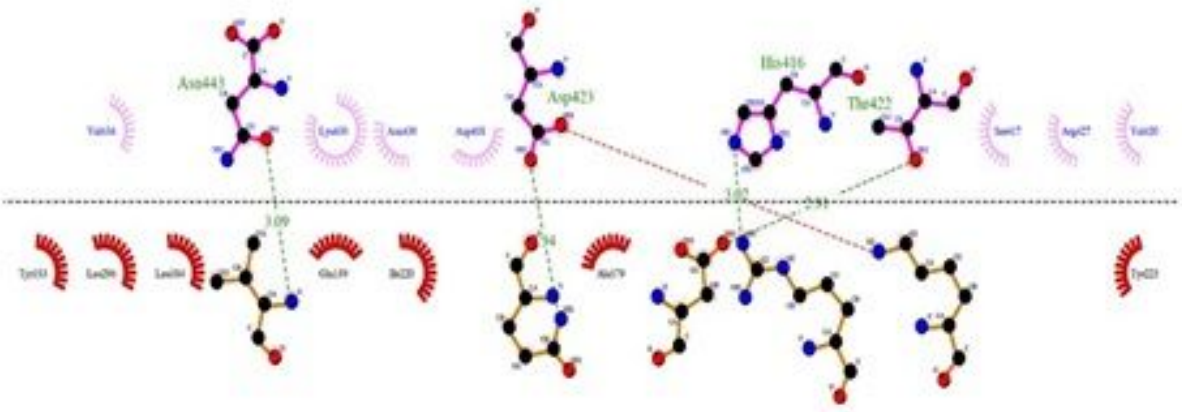

(C) Two dimensional representation of interaction study

Figure 3

Three-dimensional structure of the complex between Nsp16 (blue) and aviptadil (red). Yellow dashes indicate hydrogen bonds between respective atoms of inter-chains.

\section{Supplementary Files}

This is a list of supplementary files associated with this preprint. Click to download.

- Table1.doc 